\title{
Understanding Exercise Behavior and Dropout through Metamotivational Dominance, Exercise Identity, and Motives
}

\author{
Rachel Rahman \\ Aberystwyth University
}

\author{
Joanne Hudson \\ Swansea University
}

\author{
Stuart W. Flint \\ Leeds Beckett University
}

\begin{abstract}
Despite literature exploring interventions and strategies to encourage exercise adoption and maintenance, the dropout rate of irregular exercisers, particularly within the first six months of adoption, continues to reduce the effectiveness of such interventions. Whilst a body of literature exists exploring the dropout profile of clinical patients, less is known about the psychological and theoretical differences that discriminate exercise behavior and that could be indicative of susceptibility to dropout in the general population. Our study examines whether the metamotivational constructs of reversal theory (Apter, 1989), exercise motives, and exercise identity can discriminate between males' and females' exercise behavior, defined in relation to length of exercise participation, consistency (frequency of previous dropout) and the main type of exercise engaged in. We created an online survey to which 973 participants responded. We used MANOVA to determine whether exercise length, consistency, or type resulted in significant differences in levels of outcome variables. Where we identified significant effects, we employed discriminant function analysis to determine whether and how the dependent variables were able to discriminate between groupings. Results indicated that differing profiles of exercise identity, metamotivational dominance, and motives for exercise could discriminate between females and males who had been exercising for different lengths of time, with different levels of exercise consistency and differing types of main exercise. Our findings indicate that specific groupings may highlight individuals who are vulnerable to dropout so that strategies can be tailored more effectively for these individuals and support more appropriate strategies to develop internalized motivation.
\end{abstract}

Keywords: exercise identity, exercise motives, reversal theory, exercise, exercise dropout

The relationship between physical activity and positive health and well-being is well established (Barnes, 2010; Ekelund et al., 2016). The past decade has seen a dramatic rise in chronic illness associated with an inactive lifestyle in western societies (Ding et al., 2016; World Health Organisation, 2015) and as such, the need to encourage exercise adoption and maintain physical activity remains. However, despite a range of literature exploring interventions and strategies to encourage exercise adoption and maintenance, the dropout rate of irregular exercisers, particularly within

Rachel Rahman, Psychology Department, Aberystwyth University; Joanne Hudson, School of Sport and Exercise Sciences, College of Engineering, Bay Campus, Swansea University; Stuart W. Flint, Leeds Beckett University.

Correspondence concerning this article should be addressed to Rachel Rahman, Department of Psychology, Aberystwyth University, Penbryn 5, Penglais Campus, Aberystwyth, Ceredigion, SY23 3UX, UK. E-mail: rjr@aber.ac.uk the first 6 months of adoption, continues to reduce the effectiveness of such interventions for sustained improvements to health (James et al., 2008).

Whilst focusing on physically inactive individuals and seeking ways to encourage exercise adoption is beneficial, a significant amount could be learnt from exploring the factors that determine dropout versus long-term exercise participation to allow more targeted strategies to support those most at risk of dropping out. A number of studies have explored the determinants of dropout, attendance and/or adherence to clinical or structured interventions such as cardiac rehabilitation (Yohannes, Yalfani, Doherty, \& Bundy, 2010), programs for patients with cancer (Shang, Wenzel, Krumm, Griffith, \& Stewart, 2012) or for specific groups such as older adults (Hawley-Hague et al., 2013) and individuals who are overweight or obese (Hadžiabdić et al., 2015). This research has tended to examine the influence of demographic variables such as education level, age, and gender, as well as the role of specific health perceptions. This focus on clinical populations is beneficial for understanding determinants in specific 
populations and for accounting for individual differences to enhance the effectiveness of structured interventions, such as exercise referral or rehabilitation, that are used to encourage exercise adoption. However, limited interventions exist to support the general, otherwise healthy, public to adopt and maintain physical activity. Although numerous public health campaigns exist aimed at encouraging physical activity in the general population, these focus on a one size fits all approach, which is counterintuitive given the importance of individual differences in exercise and other health behaviors (e.g., Rose \& Parfitt, 2007). Thus, whilst profiling individuals in relation to their demographic characteristics can be useful, more consideration needs to be given to the psychological differences that discriminate varying engagement with exercise.

Although research has examined numerous predictors of, and barriers to, exercise adoption (e.g., Herring, Sailors, \& Bray, 2014; Withall, Jago, \& Fox, 2011), limited research has explored the influential factors that might determine exercise dropout in the general public. This is important to understand because intervening with this population to support exercise adherence at an early stage of adoption could prevent high levels of dropout and thus the continuous rise in chronic disease diagnoses related to physical inactivity. In addition, support at an earlier stage of exercise adoption may mean that individuals who are later required to attend a clinical rehabilitation program are already more prepared to adhere to a program of exercise, having potentially developed a new identity as an exerciser prior to referral.

There is extensive research linking exercise identity with exercise behavior, demonstrating relationships between exercise identity and exercise amount and adherence to exercise in groups of people with chronic illness (e.g., Anderson \& Cychosz, 1995; MacPherson, Kerr, \& Stirling, 2016; Pentecost \& Taket, 2011; Reifsteck, Gill, \& Labban, 2016). Research highlighting the importance of exercise identity for exercise adherence has also demonstrated gender differences, where males expressed a desire to maintain a sporty or active identity, whilst women's exercise identity was more related to health or well-being (Pentecost \& Taket, 2011).

Motivation has also been consistently highlighted as important for exercise engagement; specifically, intrinsic exercise motivation is considered to be beneficial for exercise participation and adherence and is associated with greater effort, persistence, and performance (Vansteenkiste, Simons, Lens, Sheldon, \& Deci, 2004). However, it is also known that extrinsic motives are more often associated with exerciserelated behaviors (e.g., Kilpatrick, Herbert, \& Bartholomew, 2005). Similar to exercise identities, gender differences in motives to exercise have also been reported with research suggesting that females cite more extrinsic factors such as weight management or health and males more intrinsic motives such as competition and challenge (Egli, Bland, Melton,
\& Chzech, 2011; Morris, Clayton, Power, \& Han, 1995). More recently, Molanorouzi, Khoo, and Morris (2015) considered how motives for participation in physical activity could discriminate individuals based on gender and exercise type. Notably, motives discriminating between males and females included competition, appearance, physical condition, and mastery. Motives also discriminated between the types of exercise that individuals engaged in showing that those who engaged in team sports were effectively discriminated by the motives for affiliation and mastery, those in individual racing sports (such as swimming or running) were discriminated by motives for enjoyment and lower motives for affiliation and appearance, whilst those for racquet sports were discriminated by high motives for mastery and competition. Finally, exercisers were discriminated by motives for psychological and physical condition and appearance and lower motives for mastery and competition. This may be important when considering dropout because understanding the types of activities that are chosen in relation to adaptive and maladaptive motives to exercise could inform appropriate interventions for individuals at different stages of exercise uptake.

Researchers have also explored the role of personality in predicting the uptake of exercise behavior and as a potential explanation for the discrepancy in intention to act and subsequent behavior evident when most behavior change models are employed (MacCann, Todd, Mullan, \& Roberts, 2015). These authors found that, of the personality traits measured, lower emotionality significantly predicted intention, and lower honesty-humility significantly predicted actual behavior. However, a small effect size was reported for both of these relationships. Similarly, Ingledew and Markland (2008) identified positive relationships between neuroticism and external regulation and between openness to experience and health and fitness motives, as well as negative relationships between conscientiousness, appearance, and weight motives and external and introjected regulation (a form of extrinsic regulation where the individual is regulated to avoid feelings of guilt or shame). In a later systematic review, Wilson and Dishman (2015) also identified significant relationships between neuroticism, extraversion, conscientiousness, and openness and physical activity, but with low effect sizes for all variables. This clearly demonstrates that individual differences in personality can account for differing motives to participate in a specific behavior as well as the behavioral regulation underpinning this participation. However, a large amount of the variance in motives and behavioral regulation remained unexplained in these models. This suggests that personality only accounts for a small amount of the variance or that the previous theories of personality utilised do not provide a sufficiently comprehensive framework in this context.

There are a number of models that have been tested in relation to exercise motivation, including reversal theory 
(Apter, 1989), which provides a theoretical framework that aims to account for the complexity and multidimensionality of personality. The theory proposes that an individual's personality can be described by their frequency or tendency to experience their motivation in a particular way, known as metamotivational dominance. Four dominance dimensions exist: telic-paratelic, negativistic-conformist, masterysympathy, and autic-alloic. The preferred behaviors reported by individuals who are telic dominant are serious and goalorientated while paratelic dominant individuals prefer playful, sensation orientated, and impulsive behaviors. Conformists prefer to adhere to rules, expectations, and norms, whilst negativistic dominant individuals tend to rebel against these rules, expectations, and norms. Mastery dominant individuals prefer to feel in control, strong, and tough, whereas sympathy dominance is associated with a preference for cooperation, nurturing, and tenderness. Finally, autic dominance is characterised by a focus on oneself and meeting one's own needs and alloic dominance by focusing on, and giving to, others. Combinations of dominances from different pairs are possible (e.g., telic-autic-conformist-mastery). As a result, reversal theory provides a better approach than other personality theories because it offers a parsimonious, contextual, and flexible explanation for exploring the complexity of personality.

Lindner and Kerr (2000) examined exercise motivation in relation to the metamotivational constructs of reversal theory and reported that the principal reasons for sport participation included fitness and fun, which are telic and paratelic motives, respectively. They also identified that individuals who regularly participated in exercise were most likely to report telic and alloic orientations, suggesting that telic motives supported the dedication necessary to develop fitness whilst alloic orientation demonstrated the importance of friends and social groups for those who were longer term exercisers. Non-exercisers were most likely to report paratelic, mastery, and autic orientations. Those reporting paratelic orientations indicated preferences for "other activities" implying that exercise was not considered a fun activity for this group. Mastery and autic orientation was a key determinant of nonadherence, with those who considered themselves lacking in exercise ability not wishing to engage to avoid experiencing a lack of mastery in this context. In a recent systematic review, Hudson, Males, and Kerr (2015) also identified how those with telic dominance tended to show preference for low-risk endurance sports in comparison to paratelic dominant individuals who were more likely to engage in highrisk explosive sport. Similarly, Sit, Kerr, and Wong (2008) reported that participants' motivation towards sport and exercise was comprised of telic, conformist, alloic, and sympathy styles. This evidence illustrates the relevance of reversal theory for understanding exercise behavior. However, this research remains limited as a dichotomous approach was adopted to participation, describing motivational orientations in relation to participation or non-participation. We do not manage complex behaviors, such as exercise, in this simplistic way. This is recognised not only in reversal theory but also in behavior change models such as the transtheoretical model (Prochaska \& DiClemente, 1984), where behavior is proposed to progress and regress through stages representing different degrees of consistency of behavior and psychological states. Thus, when examining exercise behavior, we need to adopt a more differentiated and dynamic approach to defining participation to account for behavioral consistency and inconsistency, which is the aim of the present study. Given the importance of exercise identity for determining exercise behavior, we also examine its role alongside motivational variables in predicting exercise behavior.

The current study therefore examines whether the metamotivational constructs of Reversal Theory (Apter, 1989), exercise motives, and exercise identity can discriminate between males' and females' exercise behavior, defined in relation to length, consistency (frequency of dropout), and main type of exercise participation. In line with previous research, we hypothesised that:

1. Longer and more consistent exercise participation will be characterised by a stronger exercise identity, more intrinsic motives for participation, and telic, conformist, alloic, and sympathy dominances.

2. Profiles of shorter, less consistent exercise participation (and thus more vulnerable to dropout) will be characterised by weaker exercise identity, more extrinsic motives, and paratelic, mastery, and autic dominances.

3. Individuals whose main type of activity is sports and group exercise are more likely to be characterised by paratelic and alloic dominance whilst those in more individual and gym-based exercise will be characterised by more telic and autic dominance.

\section{Method}

\section{Participants}

Participants were 973 individuals (65.4\% female) aged 16 to 74 years with a mean age of $33.7 \pm 13.9$ years all residing in the UK. There were no specific inclusion criteria other than being over the age of 16 , as the questionnaire was designed to be relevant for both exercisers and non-exercisers. They responded to an email invitation to participate in an online study. Of the responses, 33\% were students, $21 \%$ worked within the education sector, $14 \%$ in administration, $11 \%$ in science, $9 \%$ in management and business, $7 \%$ were unemployed, $3 \%$ worked in healthcare, and 3\% in sales, providing a cross section of the UK population. Table 1 shows the frequency of individuals represented in each category of the grouping variables for exercise length, consistency, and type of exercise. The majority of individuals were long-term 
Table 1

Frequency of Participants Representing Each Category of the Grouping Variables

\begin{tabular}{llrr}
\hline Variable & Grouping & Frequency & $(\%)$ \\
\hline Exercise length & $>10$ years & 437 & $(44.9 \%)$ \\
& $5-10$ years & 199 & $(20.5 \%)$ \\
& $1-5$ years & 223 & $(22.9 \%)$ \\
& 6 months-1 year & 45 & $(4.7 \%)$ \\
& $1-5$ months & 50 & $(5.1 \%)$ \\
& $>1$ month & 19 & $(2.0 \%)$ \\
Exercise consistency & Drop out: never & 349 & $(25.9 \%)$ \\
& Drop out: once & 294 & $(30.2 \%)$ \\
& Drop out: more than once & 218 & $(22.4 \%)$ \\
& Drop out: numerous & 112 & $(11.5 \%)$ \\
Exercise type & Gym & 180 & $(18.5 \%)$ \\
& Class & 87 & $(8.9 \%)$ \\
& High intensity sport (e.g., football) & 164 & $(16.9 \%)$ \\
& Low intensity sport (e.g., golf) & 22 & $(2.3 \%)$ \\
& Individual (e.g., running) & 520 & $(53.4 \%)$ \\
\hline
\end{tabular}

Drop out: never = "I have not dropped out for longer than 4 weeks."

Drop out: once $=$ "I have not dropped out for longer than 4 weeks on more than one occasion."

Drop out: more than once = "I have dropped out for longer than 4 weeks on more than one occasion."

Drop out: numerous = "I have dropped out for longer than 4 weeks on numerous occasions."

exercisers having been exercising for over 10 years. Other categories were well represented except for those exercising for less than one month, which is to be expected given the potential for sampling bias of those more engaged in exercise. Consideration was given to collapsing certain groups; however, given the relevance of the groupings of short duration to the likelihood of dropout, the decision was made to retain these durations in the analysis to determine if these resulted in specific patterns of motives, identity, and/or metamotivational dominance. In terms of exercise consistency, all groups were relatively evenly represented. Similarly for exercise type, all categories were well represented except for low intensity sport, which had lower numbers (See Table 1).

\section{Measures}

Personality was measured via metamotivational dominance as described in Reversal Theory and using the Motivational Style Profile in relation to sport and exercise (MSP; Apter, Mallows, \& Williams, 1998; modified by Kerr, Au, \& Lindner, 2004; MSP-SE). This is a 40-item scale using a Likert type response scale, which measures the degree to which an individual is dominant in the four metamotivational dominances: telic, negativistic, autic, and mastery. Apter et al. (1998) have demonstrated that the MSP has acceptable validity, test-retest reliability, and internal consistency, for instance, Cronbach's alpha coefficients ranged from .68 to .89 and test-retest correlations from .71 to .92 .

Exercise motives were assessed via the Exercise Motivation Inventory-2 (EMI-2; Markland \& Ingledew, 1997).
The EMI-2 includes 51 items that comprise 14 subscales assessing motives for Stress Management, Revitalisation, Enjoyment, Challenge (Psychological Motives), Competition, Social Recognition, Affiliation, Health Pressures, Ill Health Avoidance, Positive Health, Weight Management, Appearance, Strength, and Endurance, and Nimbleness. Responses are provided on a 6 point Likert type scale, anchored by 0 (not at all true for me) and 5 (very true for me). The EMI2 has shown good reliability with Cronbach's alpha coefficients ranging from .66 to .86 (Markland \& Ingledew, 1997).

Exercise identity was measured using the Exercise Identity Scale (Anderson \& Cychosz, 1994). The nine item scale measures the extent to which exercise forms part of an individual's self-concept, responded to on a Likert scale ranging from 1 "strongly disagree" to 7 "strongly agree". Anderson and Cychosz (1994) have demonstrated good test-retest reliability of the scale $(\alpha=.93)$ and internal consistency with factor loadings between .62-.91.

Exercise behavior was assessed by questioning participants on whether or not they were currently engaged in exercise with a categorical response of yes or no.

Exercise length was measured by asking how long participants had been engaged in their main exercise. These were later categorised into those who had exercised for greater than 10 years, 5-10 years, 1-5 years, 6 months- 1 year, 16 months, and less than 1 month. The final two categories were included due to theoretical proposals that these time points are critical for early adoption and potential vulnerability to dropout (1 month) and likelihood to maintain behav- 
ior (maintenance stage post 6 months of Stages of Change; Prochaska \& DiClemente, 1982).

Exercise consistency was measured by asking participants how consistent they considered their exercise behavior to have been in the past year (excluding reasons out of their control such as illness or injury). The monthly interval proposed by the Stages of Change (Prochaska \& DiClemente, 1982) between the Preparation / Action phases was used to indicate this as an appropriate timeframe that individuals might dropout but with the potential to re-engage rather than defining dropout on a more permanent basis. Thus, four categorical responses were available including, "I have dropped out for longer than 4 weeks on numerous occasions", "I have dropped out for longer than 4 weeks on more than one occasion", "I have not dropped out for longer than 4 weeks on more than one occasion", and "I have not dropped out for longer than 4 weeks".

Exercise type was assessed by asking people to identify the type of exercise that they did most regularly or would choose to do most regularly if they were exercising. Options included attending a gym, individual unstructured exercise (e.g., aerobics, circuit classes), low intensity sport (e.g., golf), high intensity sport (e.g., badminton), or an "other" category.

\section{Procedure}

Ethical approval for the study was granted by the institutional ethics committee in accordance with British Psychological Society ethical guidelines. Participants were contacted via an email campaign and asked whether they would be interested in completing an online survey about exercise motives and behavior. Interested parties were asked to follow a link to the online questionnaire where all study information was provided. Informed consent was assumed by completion and submission of the questionnaire, as explained in the study information.

\section{Data Analysis}

Data were screened for parametric assumptions and due to uneven sample sizes in the grouping conditions, homogeneity of variance was violated in several of the male and female variables. Multivariate ANOVA of male and female data (using the Wilk's Lambda test statistic due to its ability to be robust against violations of homogeneity) was initially used to determine whether the grouping variables of exercise length, exercise consistency, and exercise type resulted in significant differences in levels of metamotivational dominance, exercise motives, and exercise identity. Where significant effects were identified, discriminant function analysis was employed to determine whether and how the dependent variables were able to discriminate between the groupings. This was used as opposed to the usual post hoc tests, given that Discriminant Analysis can explore relationships beyond the linear combinations of MANOVA.

\section{Results}

\section{Descriptive Statistics}

Tables 2 through 7 show the descriptive statistics of the variables included in the analyses for males and females, respectively. Coefficients of reliability for all variables were over .68 indicating good internal consistency except for the motive of health pressures with an alpha reliability of .66 .

\section{Multivariate Analyses of Variance}

Two multivariate analyses of variance (MANOVA) were conducted (one for males and one for females) to explore the effect of exercise length, consistency, and type on metamotivational dominance, exercise identity, and exercise motives.

For males, the MANOVA indicated no significant effect of exercise length $(F(57,764)=1.247, p=.06)$, however, this was approaching significance. There was a significant effect of exercise consistency $(F(57,764)=1.612, p=.004)$ and exercise type $(F(76,1011)=1.592, p=.001)$. Interactions between the grouping variables approached but did not reach statistical significance. As a result discriminant analysis was explored for exercise length, consistency, and type in male participants.

For females, there was no significant main effect for exercise consistency $(F(957,1587)=1.156, p=.201)$. However, there was a significant main effect for exercise length $(F(95,2593)=1.376, p=.01)$ and exercise type $(F(76,2098)=1.731, p<.001)$. For females there was also a significant three way interaction between the grouping variables $(F(532,8464)=1.181, p=.003)$. As such all three grouping variables were included in the discriminant analysis for females.

\section{Discriminant Analysis of Exercise Length}

For males, two discriminant functions were identified as significant $(p<.05$; see Table 8$)$. Function I accounted for $41.8 \%$ of the variance and Function II, for $24.3 \%$. Table 9 identifies the variables most strongly correlated with these two functions. Function 1 included mastery dominance, with motives for weight management negatively and ill-health avoidance positively. Function II included autic dominance (negatively), along with motives for enjoyment, revitalisation, and stress management negatively and health prevention positively.

Overall, $42 \%$ of group membership was correctly classified with correct classifications per group as follows: $>10$ years $(44.3 \%)$; $5-10$ years $(30.3 \%) ; 1-5$ years $(37.7 \%) ; 6$ months-1 year: $(57.1 \%)$; $1-6$ months $(62.5 \%)$, and $<1$ month $(100 \%)$. Group centroids for Function I indicated that the variables particularly discriminated between those who 
Table 2

Means and Standard Deviations for Exercise Length - Male

\begin{tabular}{|c|c|c|c|c|c|c|}
\hline & $<1 \mathrm{~m}$ & $1 \mathrm{~m}-6 \mathrm{~m}$ & $6 m-1 y r$ & $1-5 y r s$ & $5-10$ yrs & $>10 \mathrm{yrs}$ \\
\hline Exercise identity & $27.00(10.42)$ & 31.63 (13.77) & $38.86(14.83)$ & $43.52(13.18)$ & $43.68(13.39)$ & $43.88(1.00)$ \\
\hline Telic dominance & $-1.25(3.30)$ & $2.88(6.58)$ & $1.29(6.37)$ & $0.51(5.38)$ & $1.71(5.49)$ & $1.50(5.02)$ \\
\hline Negativistic dominance & $-3.25(4.92)$ & $-8.50(9.17)$ & $-5.21(7.06)$ & $-6.07(6.39)$ & $-6.55(5.67)$ & $-5.19(6.33)$ \\
\hline Autic dominance & $-2.38(4.48)$ & $-4.63(10.98)$ & $-2.57(3.51)$ & $-1.80(3.62)$ & $-2.65(3.63)$ & $-3.07(4.00)$ \\
\hline Mastery dominance & $0.38(2.53)$ & $-0.50(3.06)$ & $-0.29(3.56)$ & $-0.42(3.66)$ & $-0.50(2.69)$ & $0.68(2.83)$ \\
\hline Stress management & $2.38(1.38)$ & $1.63(1.00)$ & $2.89(1.55)$ & $2.79(1.47)$ & $2.76(1.52)$ & $3.04(1.29)$ \\
\hline Revitalisation & $3.00(1.28)$ & $2.13(1.13)$ & $3.02(1.29)$ & $3.45(0.92)$ & $3.36(1.14)$ & $3.55(1.10)$ \\
\hline Enjoyment & $2.63(1.16)$ & $1.97(1.51)$ & $3.13(1.42)$ & $3.49(1.27)$ & $3.42(1.24)$ & $3.52(1.29)$ \\
\hline Challenge & $2.50(1.29)$ & $1.53(1.39)$ & $2.23(1.59)$ & $2.85(1.42)$ & $2.82(1.25)$ & $2.68(1.36)$ \\
\hline Social recognition & $2.88(0.48)$ & $0.94(0.99)$ & $1.66(1.59)$ & $2.39(1.56)$ & $2.30(1.43)$ & $1.96(1.40)$ \\
\hline Affiliation & $3.44(0.90)$ & $1.31(1.73)$ & $1.18(1.50)$ & $1.97(1.60)$ & $2.22(1.60)$ & $2.24(1.56)$ \\
\hline Competition & $3.36(0.83)$ & $1.66(1.55)$ & $1.10(1.12)$ & $2.49(1.75)$ & $2.49(1.62)$ & $2.66(1.70)$ \\
\hline Health pressures & $1.00(0.27)$ & $1.96(2.01)$ & $0.71(0.94)$ & $0.85(0.92)$ & $1.03(1.1)$ & $1.10(1.06)$ \\
\hline Ill health avoidance & $3.33(1.05)$ & $3.50(1.79)$ & $2.98(1.44)$ & $2.90(1.36)$ & $3.33(1.32)$ & $3.36(1.29)$ \\
\hline Positive health & $3.58(1.07)$ & $3.75(1.02)$ & $3.71(1.66)$ & $3.98(0.96)$ & $4.04(0.96)$ & $4.02(0.95)$ \\
\hline Weight management & $2.63(1.44)$ & $3.75(1.30)$ & $3.31(1.83)$ & $3.09(1.51)$ & $2.76(1.57)$ & $2.62(1.48)$ \\
\hline Appearance & $2.94(1.30)$ & $2.59(1.66)$ & $2.79(1.51)$ & $3.25(1.05)$ & $3.10(1.17)$ & $2.66(1.33)$ \\
\hline Strength \& Endurance & $3.25(0.84)$ & $3.53(0.81)$ & $3.77(1.56)$ & $3.96(0.94)$ & $3.87(0.89)$ & $3.50(1.19)$ \\
\hline Nimbleness & $2.25(0.69)$ & $2.17(1.26)$ & $2.67(1.22)$ & $3.19(1.29)$ & $3.34(1.26)$ & $3.19(1.22)$ \\
\hline
\end{tabular}

Table 3

Means and Standard Deviations for Exercise Length - Female

\begin{tabular}{lcccccc}
\hline & $<1 \mathrm{~m}$ & $1 \mathrm{~m}-6 \mathrm{~m}$ & $6 \mathrm{~m}-1 \mathrm{yr}$ & $1-5 \mathrm{yrs}$ & $5-10 \mathrm{yrs}$ & $>10 \mathrm{yrs}$ \\
\hline Exercise identity & $19.80(8.30)$ & $27.98(11.64)$ & $32.61(12.79)$ & $38.58(14.33)$ & $41.88(14.40)$ & $39.27(15.41)$ \\
Telic dominance & $0.13(4.72)$ & $1.36(5.00)$ & $2.74(5.22)$ & $2.17(4.71)$ & $2.32(4.44)$ & $2.14(4.68)$ \\
Negativistic dominance & $-7.53(4.02)$ & $-6.95(5.84)$ & $-9.03(4.81)$ & $-6.83(5.91)$ & $-7.77(6.00)$ & $-6.97(6.08)$ \\
Autic dominance & $-2.33(4.17)$ & $-2.83(3.74)$ & $-2.77(4.00)$ & $-3.59(3.74)$ & $-3.95(3.81)$ & $-4.04(3.87)$ \\
Mastery dominance & $-1.80(2.79)$ & $-2.38(2.99)$ & $-2.34(3.50)$ & $-1.83(3.23)$ & $-1.14(3.25)$ & $-0.74(2.95)$ \\
Stress management & $2.32(1.28)$ & $2.57(1.35)$ & $2.60(1.46)$ & $3.25(1.33)$ & $3.45(1.18)$ & $3.29(1.31)$ \\
Revitalisation & $2.33(1.30)$ & $2.67(1.30)$ & $2.71(1.24)$ & $3.37(1.15)$ & $3.56(1.20)$ & $3.53(1.29)$ \\
Enjoyment & $1.93(1.42)$ & $2.33(1.46)$ & $2.40(1.33)$ & $3.14(1.40)$ & $3.37(1.42)$ & $3.24(1.50)$ \\
Challenge & $1.97(1.37)$ & $2.05(1.28)$ & $1.68(1.11)$ & $2.50(1.32)$ & $2.23(1.38)$ & $2.21(1.41)$ \\
Social recognition & $1.40(1.08)$ & $1.34(1.36)$ & $1.19(1.24)$ & $1.59(1.25)$ & $1.52(1.31)$ & $1.40(1.31)$ \\
Affiliation & $2.22(1.39)$ & $2.03(1.70)$ & $1.33(1.35)$ & $1.92(1.56)$ & $2.20(1.66)$ & $1.89(1.51)$ \\
Competition & $1.10(0.87)$ & $1.20(1.51)$ & $0.84(1.12)$ & $1.55(1.51)$ & $1.75(1.62)$ & $1.55(1.66)$ \\
Health pressures & $1.09(1.13)$ & $1.33(1.34)$ & $1.37(1.67)$ & $1.19(1.26)$ & $1.04(1.13)$ & $1.12(1.19)$ \\
Ill health avoidance & $3.40(1.89)$ & $3.13(1.27)$ & $3.41(1.17)$ & $3.42(1.14)$ & $3.50(1.22)$ & $3.50(1.20)$ \\
Positive health & $4.02(0.87)$ & $3.75(1.21)$ & $4.04(0.89)$ & $4.14(0.90)$ & $4.20(0.88)$ & $4.09(1.02)$ \\
Weight management & $3.68(1.67)$ & $3.79(1.34)$ & $4.11(1.10)$ & $3.89(1.29)$ & $3.72(1.23)$ & $3.52(1.37)$ \\
Appearance & $3.38(1.19)$ & $3.23(1.22)$ & $3.35(1.19)$ & $3.32(1.15)$ & $3.12(1.23)$ & $3.04(1.31)$ \\
Strength \& Endurance & $2.98(1.38)$ & $2.97(1.16)$ & $3.03(1.25)$ & $3.35(1.06)$ & $3.26(1.25)$ & $3.29(1.28)$ \\
Nimbleness & $3.51(1.08)$ & $3.05(1.33)$ & $3.22(1.24)$ & $3.44(1.12)$ & $3.18(1.37)$ & $3.45(1.29)$ \\
\hline
\end{tabular}

had been exercising for $>10$ years $(.385)$ and other durations (< 1 month: .147; 1-6 months: -.819; 6 months-1 year: -.989 ; $1-5$ years: -.628; 5-10 years: -.186). Variable means indicated that males who had been exercising for over 10 years were more likely to have higher levels of mastery dominance than other groups (albeit remaining at a low level), lower levels of weight management motives, and higher levels of ill-health avoidance motives than the other groups.

Group centroids for Function II primarily differentiated those who had been exercising for between 1-6 months (2.006) and all other groups (< 1 month: .873; 6 months-1 year: -.293 ; $1-5$ years: -.290 ; 5-10 years: .091 ; $>10$ years: $-.020)$. Variable means indicated that the 1-6 month group were characterised by higher levels of alloic dominance and 
Table 4

Means and Standard Deviations for Exercise Consistency - Male

\begin{tabular}{lcccc}
\hline & Never & $\begin{array}{c}\text { Drop out } \\
\text { once }\end{array}$ & $\begin{array}{c}\text { Drop out } \\
\text { more than once }\end{array}$ & $\begin{array}{c}\text { Drop out } \\
\text { numerous }\end{array}$ \\
\hline Exercise identity & $44.74(13.42)$ & $45.42(11.66)$ & $40.81(13.80)$ & $29.04(13.36)$ \\
Telic dominance & $2.10(5.19)$ & $0.82(5.31)$ & $1.09(4.85)$ & $0.74(5.83)$ \\
Negativistic dominance & $-6.01(5.53)$ & $-5.75(6.53)$ & $-5.85(6.55)$ & $-3.15(8.31)$ \\
Autic dominance & $-2.84(3.81)$ & $-2.78(4.10)$ & $-2.98(3.59)$ & $-1.87(3.25)$ \\
Mastery dominance & $0.31(2.89)$ & $0.08(3.43)$ & $-0.17(3.02)$ & $0.61(1.93)$ \\
Stress management & $2.88(1.33)$ & $2.98(1.43)$ & $3.05(1.41)$ & $2.26(1.33)$ \\
Revitalisation & $3.57(1.03)$ & $3.50(1.01)$ & $3.31(1.18)$ & $2.67(1.43)$ \\
Enjoyment & $3.60(1.23)$ & $3.45(1.27)$ & $3.42(1.25)$ & $2.46(1.52)$ \\
Challenge & $2.74(1.39)$ & $2.83(1.29)$ & $2.62(1.40)$ & $1.96(1.35)$ \\
Social recognition & $1.95(1.46)$ & $2.12(1.45)$ & $2.19(1.36)$ & $1.65(1.42)$ \\
Affiliation & $2.17(1.57)$ & $2.11(1.64)$ & $2.38(1.59)$ & $1.55(1.31)$ \\
Competition & $2.61(1.63)$ & $2.46(1.74)$ & $2.75(1.72)$ & $1.73(1.54)$ \\
Health pressures & $1.02(1.10)$ & $0.99(0.99)$ & $1.14(1.11)$ & $1.16(1.19)$ \\
Ill health avoidance & $3.19(1.38)$ & $3.47(1.15)$ & $3.09(1.46)$ & $3.09(1.40)$ \\
Positive health & $4.03(0.99)$ & $4.11(0.86)$ & $3.87(1.01)$ & $3.52(1.28)$ \\
Weight management & $2.67(1.50)$ & $3.06(1.54)$ & $2.44(1.57)$ & $2.85(1.41)$ \\
Appearance & $2.78(1.31)$ & $3.05(1.24)$ & $2.81(1.23)$ & $2.59(1.32)$ \\
Strength \& Endurance & $3.56(1.19)$ & $3.85(1.03)$ & $3.70(0.93)$ & $3.39(1.25)$ \\
Nimbleness & $3.26(1.16)$ & $3.21(1.23)$ & $3.07(1.38)$ & $2.57(1.40)$ \\
\hline
\end{tabular}

Table 5

Means and Standard Deviations for Exercise Consistency - Female

\begin{tabular}{lcccc}
\hline & Never & $\begin{array}{c}\text { Drop out } \\
\text { once }\end{array}$ & $\begin{array}{c}\text { Drop out } \\
\text { more than once }\end{array}$ & $\begin{array}{c}\text { Drop out } \\
\text { numerous }\end{array}$ \\
\hline Exercise identity & $44.15(14.89)$ & $38.99(13.16)$ & $34.41(13.64)$ & $28.80(13.64)$ \\
Telic dominance & $2.60(4.79)$ & $2.22(4.62)$ & $1.91(4.46)$ & $1.13(4.90)$ \\
Negativistic dominance & $-7.64(6.04)$ & $-7.36(5.85)$ & $-6.95(6.01)$ & $-6.38(5.54)$ \\
Autic dominance & $-4.18(3.93)$ & $-3.35(3.52)$ & $-3.29(3.85)$ & $-4.28(4.12)$ \\
Mastery dominance & $-0.57(3.12)$ & $-1.78(2.92)$ & $-1.45(3.05)$ & $-1.87(3.65)$ \\
Stress management & $3.50(1.21)$ & $3.23(1.31)$ & $3.09(1.33)$ & $2.70(1.45)$ \\
Revitalisation & $3.82(1.13)$ & $3.33(1.23)$ & $3.15(1.19)$ & $2.78(1.37)$ \\
Enjoyment & $3.63(1.35)$ & $3.09(1.47)$ & $2.89(1.42)$ & $2.31(1.47)$ \\
Challenge & $2.59(1.36)$ & $2.21(1.35)$ & $2.07(1.33)$ & $1.90(1.37)$ \\
Social recognition & $1.66(1.35)$ & $1.45(1.29)$ & $1.31(1.20)$ & $1.28(1.26)$ \\
Affiliation & $2.22(1.59)$ & $2.03(1.60)$ & $1.73(1.50)$ & $1.57(1.46)$ \\
Competition & $1.90(1.76)$ & $1.48(1.53)$ & $1.29(1.38)$ & $1.16(1.40)$ \\
Health pressures & $1.12(1.23)$ & $1.03(1.08)$ & $1.35(1.37)$ & $1.07(1.24)$ \\
Ill health avoidance & $3.55(1.18)$ & $3.54(1.12)$ & $3.39(1.21)$ & $3.15(1.30)$ \\
Positive health & $4.25(0.89)$ & $4.19(0.83)$ & $4.00(0.98)$ & $3.75(1.26)$ \\
Weight management & $3.52(1.45)$ & $3.84(1.15)$ & $3.66(1.35)$ & $3.95(1.30)$ \\
Appearance & $3.04(1.32)$ & $3.33(1.17)$ & $3.11(1.17)$ & $3.21(1.34)$ \\
Strength \& Endurance & $3.48(1.25)$ & $3.22(1.16)$ & $3.16(1.17)$ & $2.97(1.26)$ \\
Nimbleness & $3.50(1.29)$ & $3.40(1.11)$ & $3.23(1.32)$ & $3.14(1.38)$ \\
\hline
\end{tabular}

Drop out: never = "I have not dropped out for longer than 4 weeks."

Drop out: once = "I have not dropped out for longer than 4 weeks on more than one occasion."

Drop out: more than once = "I have dropped out for longer than 4 weeks on more than one occasion."

Drop out: numerous = "I have dropped out for longer than 4 weeks on numerous occasions." 
Table 6

Means and Standard Deviations for Exercise Type - Male

\begin{tabular}{lccccc}
\hline & Gym & Individual & Class based & Low sport & High sport \\
\hline Exercise identity & $46.65(12.14)$ & $39.75(14.40)$ & $37.92(14.29)$ & $38.73(13.34)$ & $46.55(11.81)$ \\
Telic dominance & $1.04(4.86)$ & $2.51(5.00)$ & $1.25(4.67)$ & $1.18(6.01)$ & $-0.15(5.59)$ \\
Negativistic dominance & $-5.62(5.93)$ & $-5.46(6.22)$ & $-9.00(5.83)$ & $-6.54(6.02)$ & $-5.50(6.81)$ \\
Autic dominance & $-2.49(4.05)$ & $-3.37(3.51)$ & $-3.42(3.30)$ & $-3.18(4.05)$ & $-1.93(4.06)$ \\
Mastery dominance & $-0.18(3.40)$ & $0.30(2.83)$ & $-1.17(2.86)$ & $0.64(2.47)$ & $0.39(3.11)$ \\
Stress management & $2.88(1.58)$ & $2.83(1.26)$ & $2.42(1.55)$ & $3.07(1.28)$ & $3.03(1.40)$ \\
Revitalisation & $3.55(0.99)$ & $3.34(1.16)$ & $3.06(1.45)$ & $3.18(1.02)$ & $3.56(1.06)$ \\
Enjoyment & $3.64(1.33)$ & $3.18(1.36)$ & $3.04(1.53)$ & $3.07(1.19)$ & $3.76(1.07)$ \\
Challenge & $2.67(1.38)$ & $2.32(1.34)$ & $2.40(1.53)$ & $2.57(1.29)$ & $3.31(1.89)$ \\
Social recognition & $2.18(1.57)$ & $1.57(1.31)$ & $1.65(1.47)$ & $2.05(1.48)$ & $2.64(1.33)$ \\
Affiliation & $1.66(1.56)$ & $1.58(1.38)$ & $2.19(1.59)$ & $2.52(1.37)$ & $3.31(1.26)$ \\
Competition & $2.38(1.64)$ & $1.83(1.52)$ & $2.38(1.92)$ & $2.41(1.43)$ & $3.71(1.31)$ \\
Health pressures & $1.09(1.14)$ & $1.05(1.13)$ & $1.39(1.32)$ & $1.56(0.93)$ & $0.90(0.89)$ \\
Ill health avoidance & $3.33(1.42)$ & $3.34(1.27)$ & $3.11(1.62)$ & $3.48(0.82)$ & $3.06(1.35)$ \\
Positive health & $4.23(0.87)$ & $3.90(0.93)$ & $3.89(1.42)$ & $3.82(1.06)$ & $3.96(1.07)$ \\
Weight management & $3.18(1.50)$ & $2.77(1.49)$ & $3.08(1.52)$ & $2.36(1.43)$ & $2.49(1.57)$ \\
Appearance & $3.70(0.95)$ & $2.55(1.28)$ & $2.33(1.26)$ & $2.34(1.06)$ & $2.79(1.26)$ \\
Strength \& Endurance & $4.18(0.84)$ & $3.34(1.15)$ & $3.54(1.07)$ & $2.93(1.28)$ & $3.86(1.02)$ \\
Nimbleness & $3.21(1.31)$ & $3.08(1.27)$ & $3.14(1.26)$ & $2.91(1.28)$ & $3.27(1.18)$ \\
\hline
\end{tabular}

Table 7

Means and Standard Deviations for Exercise Type - Female

\begin{tabular}{lccccc}
\hline & Gym & Individual & Class based & Low sport & High sport \\
\hline Exercise identity & $40.33(12.38)$ & $36.33(15.58)$ & $37.09(14.74)$ & $33.64(14.00)$ & $46.09(13.74)$ \\
Telic dominance & $3.12(4.67)$ & $2.09(4.78)$ & $2.25(4.17)$ & $2.91(4.11)$ & $0.50(4.47)$ \\
Negativistic dominance & $-8.01(5.68)$ & $-6.99(6.14)$ & $-7.60(5.32)$ & $-8.00(8.99)$ & $-6.67(5.03)$ \\
Autic dominance & $-2.85(3.60)$ & $-4.19(3.99)$ & $-3.33(3.41)$ & $-2.86(4.18)$ & $-3.11(3.43)$ \\
Mastery dominance & $-2.20(3.34)$ & $-1.03(3.21)$ & $-1.95(2.55)$ & $-0.50(3.78)$ & $-0.99(2.82)$ \\
Stress management & $3.33(1.17)$ & $3.12(1.40)$ & $3.19(1.24)$ & $2.91(1.59)$ & $3.59(1.12)$ \\
Revitalisation & $3.52(1.13)$ & $3.22(1.34)$ & $3.50(1.24)$ & $3.15(1.45)$ & $3.83(0.90)$ \\
Enjoyment & $3.14(1.38)$ & $2.95(1.53)$ & $3.19(1.49)$ & $2.80(1.45)$ & $3.88(1.05)$ \\
Challenge & $2.32(1.39)$ & $2.07(1.35)$ & $2.26(1.36)$ & $2.75(0.88)$ & $3.08(1.21)$ \\
Social recognition & $1.59(1.29)$ & $1.27(1.23)$ & $1.43(1.22)$ & $1.41(1.38)$ & $1.30(1.33)$ \\
Affiliation & $1.52(1.38)$ & $1.69(1.48)$ & $2.30(1.46)$ & $2.05(1.62)$ & $3.61(1.21)$ \\
Competition & $1.31(1.44)$ & $1.25(1.40)$ & $1.40(1.40)$ & $1.98(1.76)$ & $3.36(1.61)$ \\
Health pressures & $1.20(1.32)$ & $1.14(1.21)$ & $1.40(1.36)$ & $1.03(1.18)$ & $0.82(1.00)$ \\
Ill health avoidance & $3.53(1.16)$ & $3.44(1.23)$ & $3.69(1.01)$ & $3.64(1.21)$ & $3.10(1.03)$ \\
Positive health & $4.24(0.82)$ & $3.99(1.06)$ & $4.36(0.72)$ & $4.09(1.00)$ & $4.17(0.79)$ \\
Weight management & $4.18(1.07)$ & $3.59(1.37)$ & $3.88(1.25)$ & $3.57(1.56)$ & $3.45(1.38)$ \\
Appearance & $3.64(1.10)$ & $2.99(1.29)$ & $3.51(1.04)$ & $2.91(1.07)$ & $3.01(1.15)$ \\
Strength \& Endurance & $3.34(1.11)$ & $3.14(1.29)$ & $3.54(1.07)$ & $3.55(10.11)$ & $3.42(1.11)$ \\
Nimbleness & $3.51(1.21)$ & $3.22(1.34)$ & $3.89(0.94)$ & $3.76(0.83)$ & $3.21(1.14)$ \\
\hline
\end{tabular}

motives to exercise due to health pressures with lower motives for enjoyment, revitalisation, and stress management.

Only one discriminant function was significant in the female group ( $p<.05$; see Table 8 ) and accounted for $61.2 \%$ of the variance. autic dominance was included in this function (negatively) as well as exercise identity, and, positively, motives of revitalisation, enjoyment, and stress management (see Table 9). Overall, 32.4\% of group membership was cor- rectly classified with correct classifications per group as follows: > 10 years $(28.9 \%)$; 5-10 years $(33.8 \%)$; $1-5$ years (29.0\%); 6 months-1 year $(48.4 \%)$; $1-6$ months $(35.7 \%)$, and $<1$ month $(73.3 \%)$. Group centroids indicated that this function particularly differentiated between those who had been exercising for a longer period of time (5-10 years: .352; > 10 years: .233) and those who were relatively new to exercise (6 months-1 year: -.589 ; 1-6 months: -.932 ; < 1 month: 
Table 8

Discriminant Function Analyses Results

\begin{tabular}{lllccccc}
\hline Variable & Gender & Function & Eigenvalue & Canonical Correlation & Wilk's Lambda & Chi square & df \\
\hline Exercise length & Males & I & .220 & .425 & .611 & 158.669 & 95 \\
& & II & .128 & .337 & .746 & 94.566 & 72 \\
& Females & I & .197 & .406 & .739 & 188.287 & 95 \\
Exercise consistency & Males & I & .193 & .402 & .719 & 106.877 & 57 \\
& Females & I & .211 & .418 & .762 & 169.190 & 57 \\
Exercise type & Males & I & .447 & .556 & .512 & 216.103 & 76 \\
& & II & .257 & .452 & .741 & 96.698 & 54 \\
& Females & I & .361 & .515 & .619 & 299.127 & 76 \\
& & II & .100 & .301 & .842 & 107.28 & 54 \\
\hline
\end{tabular}

$-1.704)$ with the centroid for 1-5 years siting relatively centrally (-.140). Using variable means it is evident that members of each group were more likely to be alloic dominant; however, the function analysis suggests that those classified as less alloic dominant with weaker exercise identity, lower motives for revitalisation, enjoyment, and stress management were more likely to have been exercising for under one year.

\section{Discriminant Analysis of Exercise Consistency}

For males, only one discriminant function was significant $(p<.05$; see Table 8) and accounted for $54.5 \%$ of the variance. Negativistic dominance and the motive of health pressures loaded negatively onto this function whilst exercise identity, enjoyment, revitalisation, challenge, positive health, and nimbleness all loaded positively (Table 9). Overall, $45.8 \%$ of group membership was correctly classified with correct classifications per group as follows: not dropped out (43.1\%); dropped out once (45.0\%); dropped out more than once (42.6\%), and dropped out numerous times (70.4\%). Group centroids differentiated linearly between the different groupings (not dropped out: .264; dropped out once: .104; dropped out more than once: -.242; dropped out numerous times: -1.355) but with a notable difference between the "dropped out more than once and dropped out numerous times" groups and the other groupings indicative of consistent participation. Variable means indicated that those who had dropped out numerous times had weaker exercise identity and lower levels of the identified motives than other groups. This group displayed marginally higher motives for health pressures and, whilst all group means suggested conformist dominance, participants were less conformist in the "dropped out numerous times" group than the others.

For females, only one discriminant function was significant $(p<.05$; see Table 8$)$ and accounted for $72.2 \%$ of the variance. Like the males, negativistic dominance was included in this discriminant function (negatively), with the addition of telic dominance. Exercise identity also loaded positively onto this function, along with motives for enjoy- ment, revitalisation, stress management, challenge, competition, positive health, affiliation, strength, social recognition, and nimbleness (Table 9). Overall, $43.1 \%$ of group membership was correctly classified with correct classifications per group as follows: not dropped out $(55.1 \%)$; dropped out once (35.7\%); dropped out more than once (29.9\%), and dropped out numerous times $(55.3 \%)$. The group centroids and mean scores indicated that the function discriminated between the different groups but notably between those who had not dropped out and those who had dropped out numerous times (not dropped out: .554; dropped out once: .008; dropped out more than once: -.271 ; dropped out numerous times: -.831) such that those who were less telic and conformist, with a weaker exercise identity, and lower levels of influencing motives were more likely to have dropped out on numerous occasions.

\section{Discriminant Analysis of Exercise Type}

For males, two discriminant functions were identified as significant $(p<.05$; see Table 8$)$. Function I accounted for $57.6 \%$ of the variance and Function II, for $33.1 \%$. Table 9 identifies the variables most strongly correlated with these two functions. telic dominance loaded negatively onto the first discriminant function along with positive loads for the motives of affiliation, competition, and challenge. Overall, $47.6 \%$ of group membership was correctly classified with correct classifications per group as follows: high intensity sport $(60.6 \%)$; low intensity sport $(63.6 \%)$; class attendance $(50.0 \%)$; individual exercise $(32.4 \%)$, and gym exercise $(58.1 \%)$. The group centroids indicated that Function I appeared to discriminate between the different groups, particularly between those engaging in high intensity sport and the other groups (high intensity sport: 1.031; low intensity sport: .196; class attendance: .118; individual exercise: -.403; gym exercise: -.569 ). Mean scores showed that those engaging in high intensity sport had the lowest levels of telic dominance and higher motives to exercise for challenge, affiliation, and competition in comparison to the other groups. 
Table 9

Structure Matrix by Exercise Behavior and Gender

\begin{tabular}{|c|c|c|c|c|c|c|}
\hline & \multicolumn{4}{|c|}{ Males } & \multicolumn{2}{|c|}{ Females } \\
\hline & Variable & Function I & Function II & Variable & Function I & Function II \\
\hline \multirow[t]{8}{*}{ Exercise length } & Mastery dominance & .356 & & Exercise identity & .668 & \\
\hline & Weight management & -.313 & & Revitalization & .549 & \\
\hline & Ill health avoidance & .230 & & Enjoyment & .514 & \\
\hline & Enjoyment & & -.486 & Stress management & .456 & \\
\hline & Revitalization & & -.478 & Autic dominance & -.253 & \\
\hline & Health prevention & & .422 & & & \\
\hline & Stress management & & -.372 & & & \\
\hline & Autic dominance & & -.277 & & & \\
\hline \multirow[t]{13}{*}{ Exercise consistency } & Exercise identity & .766 & & Exercise identity & .797 & \\
\hline & Enjoyment & .520 & & Enjoyment & .661 & \\
\hline & Revitalisation & .516 & & Revitalisation & .629 & \\
\hline & Challenge & .357 & & Stress management & .423 & \\
\hline & Positive health & .347 & & Challenge & .397 & \\
\hline & Nimbleness & .343 & & Competition & .379 & \\
\hline & Negativistic & -.258 & & Positive health & .365 & \\
\hline & Health pressures & -.101 & & Affiliation & .327 & \\
\hline & & & & Strength & .310 & \\
\hline & & & & Social recognition & .244 & \\
\hline & & & & Nimbleness & .222 & \\
\hline & & & & Telic & .214 & \\
\hline & & & & Negativistic & -.152 & \\
\hline \multirow[t]{14}{*}{ Exercise type } & Affiliation & .810 & & Competition & .740 & \\
\hline & Competition & .721 & & Affiliation & .700 & \\
\hline & Challenge & .415 & & Challenge & .348 & \\
\hline & Telic dominance & -.262 & & Enjoyment & .294 & \\
\hline & Appearance & & .738 & Appearance & & .665 \\
\hline & Strength & & 669 & Nimbleness & & .475 \\
\hline & Exercise identity & & .464 & Weight management & & .473 \\
\hline & Social recognition & & .389 & Mastery dominance & & -.466 \\
\hline & Enjoyment & & .337 & Autic dominance & & .431 \\
\hline & Positive health & & .259 & Positive health & & .431 \\
\hline & Autic dominance & & .216 & Revitalisation & & .367 \\
\hline & & & & Social recognition & & .363 \\
\hline & & & & Strength & & .331 \\
\hline & & & & Negativistic dominance & & -.189 \\
\hline
\end{tabular}

In the second function, autic dominance loaded positively along with exercise identity and motives for appearance, strength, social recognition, enjoyment, and positive health. The group centroids indicated that Function II appeared to discriminate between the different groups, particularly between those engaging in gym-based exercise and those in the other groups, especially low intensity sport (gym exercise: .820 , high intensity sport: .133, class attendance: -.387 ; individual exercise: -.410 ; low intensity sport -.823 ). Mean scores showed that those engaging in gym-based activities were more likely to be alloic dominant, with a stronger exercise identity and high levels of the motives outlined.

For females, two discriminant functions were identified as significant ( $p<.05$; see Table 8$)$. Function I accounted for $66.9 \%$ of the variance and Function II, for $18.5 \%$. Table 9 identifies the variables most strongly correlated with these two functions. In contrast to the males, no dominances correlated with the first function, which loaded the motives for competition, affiliation, challenge, and enjoyment. This function discriminated between the different groups but with a notable difference between the high intensity sport group and the groups engaging in class-based, individual, and gymbased exercise (high intensity sport: 1.655; low intensity sport: .298; class attendance: -.603; individual exercise: -.173 ; gym exercise: -.471 ) such that those participating in high intensity sport reported higher levels of these motives, whilst those participating in individual and gym-based exercise reported lower levels of these motives. 
In contrast, mastery dominance, negativistic dominance (both negatively), and autic dominance (positively) loaded onto the second function, along with the motives for appearance, nimbleness, weight management, positive health, revitalisation, social recognition, and strength. The group centroids indicated that Function II also systematically discriminated between the different groups, again most notably between individual exercise and gym and class attendance (gym exercise: .490; class attendance: .450; high intensity sport: .094; low intensity sport: -.079 ; individual exercise: -.242). Overall, $42.9 \%$ of group membership was correctly classified with correct classifications per group as follows: high intensity sport (74.3\%); low intensity sport (63.6\%); class attendance $(44.0 \%)$; individual exercise $(34.9 \%)$, and gym exercise (47.6\%). Variable means suggested that those primarily exercising individually reported being more autic dominant and had lower levels of all motives identified whilst those in class and gym-based activities reported higher negativistic and mastery dominance and higher levels of the motives outlined.

\section{Discussion}

The current study examined whether the metamotivational constructs of Reversal Theory (Apter, 1989), exercise identity, and exercise motives could discriminate between length, consistency, and main type of exercise behavior, and, if so, whether or not this differed in males and females. On average males and females with weaker exercise identity had been exercising for shorter lengths of time, and were more inclined to have dropped out on numerous occasions. On average females, regardless of length or consistency of exercise behavior, reported being telic, conformist, alloic, and sympathy dominant. However, males who had been exercising for less than one month reported being more paratelic and sympathy dominant in comparison to males who had been exercising for longer, who were more telic and mastery orientated. Motives of stress management, revitalisation, enjoyment, ill-health avoidance, positive health, and strength and endurance tended to increase with exercise length and consistency for both genders with some variation in the patterns seen for different motives across behavior categories and genders.

\section{Exercise Length}

For males, two functions discriminated between exercise length groups. Males who had been exercising for over 10 years were more likely to have higher levels of mastery dominance than other groups (albeit remaining at a low level), lower levels of weight management motives and higher levels of ill-health avoidance motives than the other groups. This lends partial support for hypothesis 1 that those who have been exercising for longer durations will demonstrate higher levels of intrinsic motives and lower levels of extrinsic motives. However, the finding that mastery dominance is higher in males who have been exercising for longer contradicts previous findings by Sit et al. (2008), where exercise was associated with more sympathy dominance. It should be noted however, that although the finding indicated slightly higher levels of mastery dominance, these remained at a low level, suggesting no strong preference for this dominance profile.

In the second function, males who had been exercising for between 1-6 months were characterised by higher levels of alloic dominance and motives to exercise due to health pressures but lower motives for enjoyment, revitalisation, and stress management than individuals in other groups. This supports this time frame as a vulnerable stage for dropout when males don't appear to be experiencing the positive attributes often associated with exercise. In contrast to findings by Lindner and Kerr (2000) who associate alloic dominance with regular exercisers, the findings suggest that the role of others may be key to male engagement during this early stage. It may be that males are drawing comparisons with other exercisers that motivate their engagement through competition, or perhaps, the support of others during this less enjoyable phase is critical to their continued engagement.

A significant main effect of exercise length was also identified for females with one significant discriminant function. In contrast to the male group, this suggested that females who had been exercising for under one year were alloic dominant but at a lower level than other groups (less other focused; but not autic dominant), had weaker exercise identity and weaker motives to exercise for revitalisation, enjoyment, and stress management than groups who had been exercising for over 5 years. This lends partial support for hypothesis 1, which stated that longer-term exercise participation is likely to be associated with stronger exercise identity development, echoing findings by Pentecost and Taket (2011) and Reifsteck et al. (2016). Identifying strategies to support exercise identity development in those who have been exercising for shorter periods of time and are thus, more at risk of dropout, may be an important consideration.

The role of alloic dominance for females is in line with the hypotheses and the metamotivational profiles that Lindner and Kerr (2000) associated with regular exercisers; however, the other metamotivational dominances appear to be less relevant for discriminating between groups. Given that alloic dominance indicates an individual's preference to seek a connection to others, it may be that alloic dominant individuals are attracted to a behavior such as exercise to fulfil their motives of enjoyment, stress management, and recreation given its potential for socialising with others. Fulfilment of this need through exercise may therefore explain why these individuals are likely to have engaged in exercise behaviors over longer periods of time than other groupings. 
The pattern of higher levels of intrinsic motives associated with the longer duration groupings are also interesting because they are relatively self-determined motives that do not rely on comparisons with others and as such are not likely to detract from or compete with the need to seek a connection with others in the exercise environment. It is also interesting that individuals who are "other focused" and thus, likely to put others first, are motivated to engage in exercise for more self-focused motives such as revitalisation, enjoyment, and stress management. This might suggest that exercise participation is an opportunity to nurture the self in an otherwise "other focused" individual. Additionally, perhaps it is unsurprising that these motives are associated with longer-term exercisers, given that revitalisation and stress management are benefits commonly associated with exercise. However, for those relatively new to exercise, these positive outcomes may not yet have been experienced and as such are less likely to be motivators to continue to participate. Therefore, it may be that using these as a means of encouraging exercise adoption in males and females will lead to disappointment and disillusionment when these are not immediately experienced and could lead to those in the early stages of exercise being more inclined to dropout. It is also likely that individuals new to exercise will experience some discomfort during and after exercise, such as delayed onset of muscle soreness, that they would, over time, become accustomed to, leading to an overall more positive experience (i.e., feeling more revitalized; Baird, Graham, Baker, \& Bickerstaff, 2012; Suni, Miilunpalo, Asikainen, \& Laukkanen, 1998). Thus, focusing on the opportunities to connect to others could be a more suitable approach for males and females during exercise adoption and encouraging individuals to appreciate the additional benefits associated with exercise after a longer period of participation. Further research using tailored interventions to determine whether individuals adopting exercise are more likely to continue participation if they are exercising in a group versus individually could be useful to test these suggestions.

\section{Exercise consistency}

For males, a difference was identified between the "dropped out numerous times" group and the other groupings. Variable means indicated that those who had dropped out numerous times had lower exercise identity, and lower motives for revitalization, enjoyment, challenge, positive health, and nimbleness, than all other groups. This group displayed marginally higher motives for health pressures and whilst all group means suggested conformist dominance, those in the "dropped out numerous times" group were less conformist than in other groups. Thus, overall these findings lend support to the hypotheses that exercise identity and intrinsic motives (such as revitalisation, enjoyment and positive health) would be associated with more consistent exercise behavior, whilst a weaker exercise identity and exter- nal motives would associate with more inconsistent participation. In addition, the inclusion of conformist dominance within this profile may also suggest that conformity is associated with more intrinsic motives whilst a more rebellious orientations is related to extrinsic motives and as such to greater susceptibility to dropout.

Adhering to exercise is a socially desirable behavior and as such it is understandable that those most inclined to persevere with the behavior are those who are most conformist dominant, whilst those who are regularly dropping out are likely to be less conformist dominant, especially when exercising due to health pressures. Similarly, it is logical that those with a higher exercise identity are less inclined to dropout given that the behavior is consistent with their own identity. In terms of motives, it may be that individuals who are primarily motivated to exercise because of health pressures are more likely to be under pressure from others (extrinsic) or need to exercise as a result of prior inactivity, consequently leading to health concerns. Regardless, this highlights the vulnerability of individuals exercising for this reason and also lends support to previous literature that has identified the challenge of encouraging individuals to adhere to programs of exercise in clinical contexts (e.g., Yohannes et al., 2010; Shang et al., 2012). Similar to the variable "exercise length", the motives associated with greater exercise consistency reflected exercise benefits or experiences of mastery associated with exercise that take time to develop. Thus, it is understandable that individuals who are less inclined to dropout are motivated by the positive benefits that they experience from this exercise behavior. It appears therefore that for males, ensuring strategies that help them to identify their own motivations to exercise as opposed to externalised pressures and ensuring that engagement is achievable and not overly challenging, allowing individuals to develop a sense of accomplishment relative to their performance, is of importance to supporting those most at risk of dropping out.

For females, those who were less telic and less conformist dominant with a weaker exercise identity and lower levels of influencing motives were more likely to have dropped out on numerous occasions. As with the male participants, Conformity dominance is not unexpected in this function to explain more consistent exercise behavior, as is the observation that those who are more goal orientated (telic) are also more inclined to be consistent in their behavior, with individuals who are more paratelic (spontaneous) in the groups who reported dropping-out, suggesting the potential importance of exercise being experienced as fun for this group. Again, this supports the profiles of regular exercisers identified by Lindner and Kerr (2000). However, considering exercise behavior in a more dynamic fashion has teased out the differential role of metamotivational dominances, distinguishing exercise length versus consistency. Notably, exercise identity was again the factor loading most highly to the function thus accounting 
for most of the discrimination between groups and indicating the importance of this variable for exercise persistence and consistency. It is also noteworthy that whilst a number of motives were associated with this function, all of the motives were higher in those with the most consistency in their exercise behavior and lower in those who had dropped out numerous times. Thus, rather than there being motives that are more salient to those at the stage of exercise adoption and others for those who have been exercising for longer, it appears, generally speaking, that those who are dropping out numerous times have low levels of motives for exercise across all reported motives and as such this suggests that one of the key reasons for individuals dropping out of exercise is that they see little relevance in exercising for any reason. This may indicate the importance of encouraging individuals to reflect on why they are starting a programme of exercise, in order to develop stronger motives that can then be applied when seeking the motivation to continue to exercise. Person-centred strategies such as Motivational Interviewing (Miller \& Rollnick, 2012), which acknowledge and incorporate individual motives, have demonstrated positive outcomes in many areas of behavior change (e.g., Rubak, Sandbæk, Lauritzen, Borch-Johnsen, \& Christensen, 2009) and these results may demonstrate why this strategy is likely to be more effective than more prescribed methods.

\section{Exercise Type}

For males, two discriminant functions were identified. The first was able to discriminate between those engaging in high intensity sport and the other groups such that those engaging in high intensity sport had the lowest levels of telic dominance and higher motives to exercise for challenge, affiliation, and competition in comparison to the other groups. These motives are logically associated with sporting activity suggesting the drive for a combination of affiliation with team mates and the challenge and competition associated with sport are key to participation. This profile supports previous findings by Egli et al. (2011) that these are important motives that distinguish male from female exercisers. Individuals in this group were less telic orientated than their counterparts who engaged more in individual and gymbased activities. This may be because those who are less telic dominant are more likely to be able to enjoy the high intensity of competitive sport and the physical exertion without thinking about potential negative consequences such as injury. This supports hypothesis 3 and is corroborated by Hudson et al. (2015) whose review identified that paratelic dominants showed a preference for explosive sports in comparison to telic dominants who preferred low risk endurance sport and exercise.

The second function discriminated between males whose main activity was gym based in comparison to other groups based on higher motives of appearance, strength, social recognition, enjoyment, and positive health along with a stronger exercise identity and alloic dominance. Thus in this gym-based group males were more other-focused (alloic) and were more likely to be motivated by their own physical image, seeing gym participation as an opportunity for social recognition and enjoyment. This was contrary to hypothesis 3 , which proposed that those who were more autic dominant would prefer self-focused activities such as individual exercise.

For females, two discriminant functions were identified as significant. The first function discriminated between the different groups but with a notable difference between the high intensity sport group and the groups engaging in class-based, individual and gym-based exercise. Those participating in high intensity sport reported higher levels of motives that are typically associated with sporting contexts, namely, competition, affiliation, challenge, and enjoyment, whilst those participating in individual and gym-based sports reported lower levels of these motives.

In contrast, mastery dominance, negativistic dominance, and autic dominance loaded onto the second function along with the motives for appearance, nimbleness, weight management, positive health, revitalisation, social recognition, and strength. Variable means and group centroids suggested that those exercising individually were discriminated from those exercising in classed-based and gym activities. Not surprisingly, those exercising individually were more likely to be autic dominant (self-focused) and reported lower levels of these appearance related motives whilst those exercising in classes or gyms were more likely to be negativistic and mastery dominant and reported higher levels of the motives. A couple of points are worth noting here; the first is the distinction between individual based exercise (e.g., running) and gym attendance. Although both involve exercising independently it appears that the metamotivational dominance and motives of gym exercisers align more closely with class attenders and as such may indicate that the decision to exercise amongst others engaging in a similar task is one of the key features of the decision to exercise in this context. Thus, whilst gym exercise can be engaged in independently, it appears that the role of others in this environment is an important consideration for females. Second, it is notable that women exercising amongst others (gym and classes) reported higher levels of appearance related motives such as weight management, appearance, strength, and nimbleness suggesting that drawing comparisons with others is fostered in these environments where fat burning and appearance is emphasised and is line with research by Egli et al. (2011).

Despite these promising results some limitations of the current research should be noted. Whilst the research attracted a relatively large sample size and completion from a broad demographic, the sample was predominantly made up of regular exercisers with lower numbers representing 
shorter exercise durations. As such, more research is needed to continue to explore the relevance of the identified variables to these groups to ensure generalisability of these findings. Similarly, the cross-sectional nature of this research also means that whilst trends can be identified this research cannot determine whether manipulation of these variables will result in increased length or consistency of participation for these individuals. Future research would benefit from implementing the principles of this research by using tailored interventions matched to the profiles of individuals in the vulnerable to dropout groups in order to explore these relationships further.

\section{Conclusion}

In conclusion, this research has used discriminant function analysis to consider the complexity of a combination of factors that may help to distinguish groups vulnerable to dropout, by considering the role of exercise identity, metamotivational dominance, and exercise motives to distinguish exercise length, consistency, and exercise type. Exercise identity loaded consistently as one of the stronger factors associated with exercise length and consistency in both males and females. Weak identity is therefore likely to be a critical component of vulnerability to dropout and as such how to foster a sense of exercise identity should be an important consideration in interventions to engage individuals in exercise and to support maintenance of exercise behavior in active populations.

Interestingly, three of the four metamotivational dominances contributed to distinguishing groups in slightly different ways. In females, weaker alloic dominance was more indicative of shorter exercise participation and an increased likelihood to engage in individual exercise such as running or swimming whilst for males alloic dominance characterised those who had been exercising for shorter periods and were more likely to be gym attendees. Weaker conformist and telic dominance distinguished more inconsistent exercisers with a history of multiple dropouts and were more likely to be indicative of male engagement in high intensity sport. In contrast for females, negativistic and mastery dominance were indicative of gym- and class-based exercise. The present study suggests that better consideration of the individual's own reasons for engaging in exercise behavior, encouraging spontaneous enjoyment and socialisation with others as well as less rigid rule orientated activities would be more in line with the metamotivational dominances of early adoption exercisers. However, mechanisms then need to be established to help individuals to develop a stronger exercise identity that will feed into more goal orientated and directed approaches to exercise behavior as they become adopted into the routine habit of participation. In males, health pressures also appeared to be a slightly higher motive in males who were more inclined to dropout. This raises questions regarding the approaches currently used to encourage exercise adoption through highlighting health risks of physical inactivity. Thus, as observed in research examining other health behaviors such as nutritional intake (e.g., Leikas, Lindeman, Roininen, \& Lähteenmäki, 2007) using approach-based rather than avoidance-based messages may be more beneficial in reducing exercise dropout. In addition, as suggested for females, using self-focused approaches such as motivational interviewing may support males to develop a more internalised motivation to engage in exercise activity with longer-term success.

\section{References}

Anderson, D. F., \& Cychosz, C. M. (1994). Development of an exercise identity scale. Perceptual and Motor Skills, 78(3), 747-751. doi.10.1177/003151259407800313

Anderson, D. F., \& Cychosz, C. M. (1995). Exploration of the relationship between exercise behavior and exercise identity. Journal of Sport Behavior, 18(3), 159-166.

Apter, M. J. (1989). Reversal theory: Motivation, emotion and personality. London: Routledge.

Apter, M. J., Mallows, R., \& Williams, S. (1998). The development of the motivational style profile. Personality and Individual Differences, 24, 7-18. doi 10.1016/S01918869(97)00148-7

Barnes, P. (2010). Physical activity among adults: United States, 2000 and 2005. NCHS Health E-Stat. Retrieved from http://www.cdc.gov/nchs/data/hestat/physicalactivity/ physicalactivity.htm

Baird, M. F., Graham, S. M., Baker, J. S., \& Bickerstaff, G. F. (2012). Creatine-kinase-and-exercise-related muscle damage implications for muscle performance and recovery. Journal of Nutrition and Metabolism, 2012. doi:10.1155/2012/960363

Ding, D., Lawson, K. D., Kolbe-Alexander, T. L., Finkelstein, E. A., Katzmarzyk, P. T., van Mechelen, W., \& Pratt, M. (2016). The economic burden of physical inactivity: A global analysis of major non-communicable diseases. The Lancet, 388(10051), 1311-1324. doi 10.1016/S01406736(16)30383-X.

Egli, T., Bland, H. W., Melton, B. F., Chzech, D. R. (2011). Influence of age, sex and race on college students' exercise motivation of physical activity. Journal of American College Health, 59(5), 399-406. doi: 10.1080/07448481.2010.513074.

Ekelund, U., Steene-Johannessen, J., Brown, W. J., Fagerland, M. W., Owen, N., Powell, K., et al. (2016). Does physical activity attenuate, or even eliminate, the detrimental association of sitting time with mortality? A harmonised meta-analysis of data from more than 1 million men and women. The Lancet, 388(10051), 1302-1310. doi: 10.1016/S0140-6736(16)30370-1. 
Hadžiabdić, M. O., Mucalo, I., Hrabač, P., Matić, T., Rahelić, D., \& Božikov, V. (2015). Factors predictive of drop-out and weight loss success in weight management of overweight and obese patients. Journal of Human Nutrition and Dietetics, 28(2), 24-32. doi: 10.1111/jhn.12270.

Hawley-Hague, H., Horne, M., Campbell, M., Demack, A., Skelton, D. A., \& Todd, C. (2013). Multiple levels of influence on older adults' attendance and adherence to community exercise classes. The Gerontologist, 54(4), 599-610. doi:10.1093/geront/gnt075.

Herring, M. P., Sailors, M. H., \& Bray, M. S. (2014). Genetic factors in exercise adoption, adherence and obesity. Obesity Reviews, 15(1), 29-39. doi: 10.1111/obr.12089.

Hudson, J., Males, J. R., \& Kerr, J.H. (2015). Reversal theory-based sport and exercise research: A systematic/narrative review. Psychology of Sport and Exercise, 27, 168-179.

Ingledew, D. K., \& Markland, D. (2008). The role of motives in exercise participation. Psychology $\mathcal{F}$ Health, 23(7), 807-828.doi 10.1080/08870440701405704

James, D. V. B., Johnston, L. H., Crone, D., Sidford, A. H., Gidlow, C., Morris, C., et al. (2008). Factors associated with physical activity referral uptake and participation. Journal of Sport Sciences, 26(2), 217-224. doi 10.1080/02640410701468863

Kerr, J. H., Au, C. K. F., \& Lindner, K. J. (2004). Motivation and level of risk in male and female recreational sport participation. Personality and Individual Differences, 37, 1245-1253. doi 10.1016/j.paid.2003.12.008

Kilpatrick, M., Herbert, E., \& Bartholomew, J. (2005). College students' motivation for physical activity: Differentiating men's and women's motives for sport participation and exercise. Journal of American College Health, 54(2), 87-94. doi.org/10.3200/JACH.54.2.87-94

Leikas, S., Lindeman, M., Roininen, K., \& Lähteenmäki, L. (2007). Food risk perceptions, gender, and individual differences in avoidance and approach motivation, intuitive and analytic thinking styles, and anxiety. Appetite, 48(2), 232-240.doi 10.1016/j.appet.2006.09.009

Lindner, K. J., \& Kerr, J. H. (2000). Metamotivational orientation in sport participants and non-participants. Psychology of Sport and Exercise, 1, 7-25. doi 10.1016/S14690292(00)00003-0

MacCann, C., Todd, J., Mullan, B., \& Roberts, R. D. (2015). Can personality bridge the intention behavior gap to predict who will exercise? American Journal of Health Behavior, 6, 140-147. doi 10.5993/AJHB.39.1.15

MacPherson, E., Kerr, G., \& Stirling, A. (2016). The influence of peer groups in organized sport on female adolescents' identity development. Psychology of Sport and Exercise, 23, 73-81. doi 10.1016/j.psychsport.2015.10.002

Markland, D., \& Ingledew, D. K. (1997). The measurement of exercise motives: Factorial validity and invariance across gender of a revised Exercise Motivations Inventory. British Journal of Health Psychology, 2(4), 361-376.doi 10.1111/j.2044-8287.1997.tb00549.x

Molanorouzi, K., Khoo, S., \& Morris, T. (2015). Motives for adult particpation in physical activity: Type of activity, age and gender. BMC Public Health, 15, 66-78. doi 10.1186/s12889-015-1429-7

Miller, W. R., \& Rollnick, S. (2012). Motivational interviewing: Helping people change. London, Guilford press.

Morris, T., Clayton, H., Power, H., \& Han, J. (1995). Participation motivation for different types of physical activity. In: International pre-Olympic Congress. Texas, USA: International Council of Sport Science and Physical Education.

Pentecost, C., \& Taket, A. (2011). Understanding exercise uptake and adherence for people with chronic conditions: A new model demonstrating the importance of exercise identity, benefits of attending and support. Health Education Research, 26(5), 908-922.doi 10.1093/her/cyr052

Prochaska, J. O., \& DiClemente, C. C. (1982). Transtheoretical therapy: Toward a more integrative model of change Psychotherapy: Theory, Research and Practice, 19(3), 276-288. doi 10.1037/h0088437

Prochaska, J. O., \& DiClemente, C. C. (1984). The transtheoretical approach: Crossing the traditional boundaries of therapy. Melbourne, Florida: Krieger Publishing Company. ISBN: 13: 978-0894648489.

Reifsteck, E. J., Gill, D. L., \& Labban, J. D. (2016). “Athletes" and "exercisers": Understanding identity, motivation, and physical activity participation in former college athletes. Sport, Exercise, and Performance Psychology, 5(1), 25-38.

Rose, E. A., \& Parfitt, G. (2007). A quantitative analysis and qualitative explanation of the individual differences in affective responses to prescribed and self-selected exercise intensities. Journal of Sport and Exercise Psychology, 29(3), 281-309.

Rubak, S., Sandbæk, A., Lauritzen, T., Borch-Johnsen, K., \& Christensen, B. (2009). General practitioners trained in motivational interviewing can positively affect the attitude to behavior change in people with type 2 diabetes: One year follow-up of an RCT, ADDITION Denmark. Scandinavian Journal of Primary Health Care, 27(3), 172-179. doi 10.1080/02813430903072876

Sit, C. H. P., Kerr, J. H., \& Wong, I. T. F. (2008). Motives for and barriers to physical activity participation in middleaged Chinese women. Psychology of Sport and Exercise, 9, 266-283. doi 10.1016/j.psychsport.2007.04.006

Shang, J., Wenzel, J., Krumm, S., Griffith, K., \& Stewart, K. (2012). Who will drop-out, who will drop-in, exercise adherence in a RCT among patients receiving active cancer treatment. Cancer Nurse, 35(4), 312-322. doi 10.1097/NCC.0b013e318236a3b3. 
Suni, J. H., Miilunpalo, S. I., Asikainen, T. M., \& Laukkanen, R. T. (1998). Safety and feasibility of a health-related fitness test battery for adults. Physical Therapy, 78(2), 134.

Vansteenkiste, M., Simons, J., Lens, W., Sheldon, K. M., \& Deci, E. L. (2004). Motivating learning, performance, and persistence: The synergistic effects of intrinsic goal contents and autonomy-supportive contexts. Journal of Personality and Social Psychology, 87(2), 246260. http://dx.doi.org/10.1037/0022-3514.87.2.246 doi 10.1037/0022-3514.87.2.246

Wilson, K. E., \& Dishman, R. K. (2015). Personality and physical activity: A systematic review and meta-analysis. Personality and Individual Differences, 72, 230-242.
Withall, J., Jago, R., \& Fox, K. R. (2011). Why some do but most don't. Barriers and enablers to engaging low-income groups in physical activity programmes: A mixed methods study. BMC Public Health, 11(1), 507. doi 10.1186/14712458-11-507

World Health Organisation (2015). Non-Communicable Diseases. Accessed December 12th 2016 from http:// www.who.int/mediacentre/factsheets/fs355/en/

Yohannes, A. M., Yalfani, A., Doherty, P., \& Bundy, C. (2010). Predictons of drop-out from an outpatient cardiac rehabilitation proramme. Clinical Rehabilitation, 21(3), 222-229. doi 10.1177/0269215506070771 